\title{
Cuckoo searching optimal composition of multicomponent alloys by molecular simulations
}

\author{
Aayush Sharma ${ }^{1}$, Rahul Singh ${ }^{1}$, Peter K. Liaw ${ }^{2}$ and Ganesh Balasubramanian ${ }^{1,3, a}$ \\ ${ }^{1}$ Department of Mechanical Engineering, Iowa State University, Ames, IA 50011, USA \\ ${ }^{2}$ Department of Materials Science \& Engineering, University of Tennessee, Knoxville, TN \\ 37996, USA \\ ${ }^{3}$ Microelectronics Research Center, Iowa State University, Ames, Iowa 50011, USA
}

\begin{abstract}
A robust computational design framework that couples the metaheuristic cuckoo search technique with classical molecular dynamics simulations is employed to optimize the composition of multicomponent alloys for increased tensile strength. Model binary, ternary, and quinary multi-principal element alloys are chosen as test beds to predict the influence of atomic concentration of one constituent element (design variable) on the ultimate tensile strength (objective function) of the material. The design solutions that correlate the elemental atomic fraction to strength over a number of computational search cycles are in qualitative agreement with earlier experiments. The predictive scheme explores a vast materials landscape and accelerates the elemental selection for discovery of novel multicomponent alloys.
\end{abstract}

Keywords: cuckoo search, molecular dynamics, metaheuristic optimization, highentropy alloy, ultimate tensile strength, multicomponent alloy

${ }^{a}$ Corresponding author. Address: 2092 Black Engineering, Ames, IA 50011, USA.

Phone: 515-294-9226. Email: bganesh@iastate.edu 
High-entropy alloys (HEAs), a subset of multicomponent alloys, are typically concentrated solid solutions composed of five or more principal elements, each occupying between 5 - 35 atomic \% [1-3]. The different HEA compositions that have been explored over the last decade show unique micro/nanostructures and adjustable mechanical properties [4-7]. While the theories explaining the strengthening mechanisms are still in the early stages of development, few design strategies have been proposed for optimizing elemental concentrations to achieve targeted phase and material properties [8-10]. Some of these approaches include combinatorial material synthesis, numerical schemes using ab-initio and molecular simulations, thermodynamics based calculation of phase diagrams, finite element modeling, and the Taguchi method [11-17]. The conventional methods, which involve characterization of microstructures followed by dynamic measurement of the material properties, for discovering novel multicomponent alloys are time and resource intensive. The advantage of a computational framework, on the other hand, lies in the vastness of the parameter space available for examination, not possible otherwise using only thermodynamic phase analyses or high throughput synthesis. Thus, to design novel multicomponent alloys with optimal material compositions for desired microstructures and properties, we present a simulation-driven paradigm that integrates a metaheuristic optimization technique with atomistic computations, and offers recommendations in agreement with established experimental results.

Metaheuristic algorithms enable a nature-inspired generalized optimization scheme to rapidly derive approximate solutions for intractable or gradient free problems [18-20]. Genetic Algorithm (GA) and Particle Swarm Optimization (PSO) are the most popular evolutionary algorithms with several applications in manufacturing, quality control, production, and design [21-29]. However, the effectiveness of a recent technique called cuckoo search (CS) for multi-modal design applications [30-33], and its superiority in benchmark comparisons [34, 35] against PSO and GA makes it an intelligent choice for designing multi-element HEAs. CS is a search method that imitates obligate brood parasitism of some female cuckoo species specializing in mimicking the color and pattern of few chosen host birds. The parasitic cuckoo often chooses a nest where the host has just laid its own eggs so that when the first cuckoo chick hatches, it evicts the host eggs out of the nest to increase its own food share. Specifically, from an optimization standpoint, CS (i) guarantees global convergence, 
(ii) has local and global search capabilities controlled via a switching parameter $\left(\mathrm{p}_{\mathrm{a}}\right)$, and (iii) uses Levy flights rather than standard random walks to scan the design space more efficiently than the simple Gaussian process $[32,36]$.

We integrate the CS mathematical framework with an atomistic simulation tool, molecular dynamics (MD) in this case, to optimize the elemental composition for a set of model quinary alloy with targeted properties. Since analyses using phase diagrams require extensive thermodynamic data and computationally expensive ab-initio calculations are limited to systems of a few hundred atoms only, MD simulations are chosen as the scientific probe. We construct, verify, and implement a CS-MD coupled computational design framework and demonstrate its performance to tailor the quinary $\mathrm{AlCrCoFeNi}$ alloy composition for increasing the tensile strength.

The CS optimization procedure is based on the algorithm proposed by Yang and Deb [34]. The CS implementation considers that each egg in a nest represents a solution governed by the following three idealized rules:

1. At a time, each cuckoo lays one egg and dumps it in a randomly-chosen nest.

2. Only the best nest with highest quality eggs is carried over to the next generation.

3. The probability that the host bird discovers the cuckoo egg is $p_{a} \varepsilon(0,1)$ for a fixed number of available host nests. If/when discovered, the host bird can either get rid of the cuckoo egg or build a completely new nest.

Cuckoo search possesses the advantage of a balanced combination of both the localrandom walk and the global-explorative random walk. The switching parameter, $\mathrm{p}_{\mathrm{a}}$, controls the selection between these two walks. A local random walk is represented as $x_{i}^{t+1}=x_{i}^{t}+\alpha s \otimes H\left(p_{a}-\varepsilon\right) \otimes\left(x_{j}^{t}-x_{k}^{t}\right)=0$ where, $\varepsilon=$ random number, $H(u)=$ Heaviside function, $s=$ step-size, $x_{i}^{t} \& x_{k}^{t}=$ two different solutions selected randomly by random permutation, $\otimes=$ entry wise product. A global random walk or Levy Flights is represented as $x_{i}^{t+1}=x_{i}^{t}+\alpha L(s, \lambda), \quad$ where $L(s, \lambda)=\frac{\lambda \Gamma(\lambda) \sin \left(\frac{\pi \lambda}{2}\right)}{\pi} \frac{1}{s^{1+\lambda}},\left(s \gg s_{0} \gg 0\right)$ and $>0$ is the step size scaling factor related to the scale of the problem. We verify the CS optimization code by analyzing its performance by two standard tests, the 6-hump Camel back and the Ackley function, similar to the original algorithm [34].

The convergence rate in CS optimization has been often found to be least dependent on the choice of key parameters like number of nests $(n)$ and switching parameter $\left(\mathrm{p}_{\mathrm{a}}\right)$ 
[32,36]. Our initial trials on test function like Ackley and 6-hump Camel back, revealed 0.2 to be an appropriate choice for most scenarios at different values of $n$ ( 5 to 100). This approach ensured local search to consume around $1 / 5^{\text {th }}$ of the total time, meriting suitable exploration of the global design space within reasonable computing times. Thus, for all simulations of the multi-component alloys under CS-MD framework, we use a value of $p_{a}$ equal 0.2. An efficient heuristic optimization framework ensures that the system is not trapped within any local optimum in the design/objective function landscape. CS optimization includes both the local-random walk and the global-explorative random walk with Lévy flights. Thus, a guaranteed global convergence is an intrinsic feature and a unique advantage of the CS framework [30-33]. We believe that a comparison of computational times between CS and just the local search would suggest that since the local search explorations could be stuck in local optimum, they will consume more computational resources/time (although we did not run independent simulations). In relation to this, CS would certainly be more efficient in exploring desired maxima/minima at both local and global search gradients [34,35].

The modified CS algorithm implemented here is shown in Fig. 1a, which is adapted from a generalized description of the standard CS technique [34]. The CS-MD framework, illustrated in Fig. 1b, involves optimizing user-declared design variables, such as the elemental concentration in the alloy for the desired property, the ultimate tensile strength in this case. Each cycle (generation) of CS involves comparing different solutions (nests) to retain the best candidate while replacing all unfavorable solutions with newer alternatives predicted via the global and local exploratory walks in the design space. This strategy ensures that all possible solutions are explored, and we overcome the barriers imposed by the local maxima/minima to arrive at globallyoptimum results. For every cycle, only the alloy with the elemental concentration that yields the maximum ultimate tensile strength is retained amongst all the available solutions, referred as the best nest/solution $\left(\mathrm{g}^{*}\right)$.

[Figure 1]

FIG.1. Cuckoo search (CS) and the implemented CS-MD algorithm: (a) Flowchart describing the steps in a standard CS optimization algorithm; (b) The proposed CSMD framework for the design of multicomponent alloys. 
The CS-MD framework is tested for robustness by varying input concentrations from $2.5 \%$ to $97.5 \%$, in increments of $2.5 \%$. A single MD-output file was used as the 'output' for all concentrations. The code provided the correct elemental concentrations (input) and calculated the maximum strength as equivalent for all cases tested. The implementation of multiple input parameters in this framework is a natural extension of this predictive scheme.

All the atomistic simulations of the binary, ternary subsets, and the quinary alloy are performed with LAMMPS [37]. In a 2.0 × 2.0 x $2.0 \mathrm{~nm}$ FCC lattice, elements (Al-Cr-Co-Fe-Ni) were randomly arranged to form the alloy system of 32,000 atoms, having periodic boundary conditions imposed in all directions. Energy minimization is carried out, using the conjugate gradient algorithm with energy and force tolerance set to $10^{-15}$ units. First initialization at 2,200 K under an isothermalisobaric (NPT) ensemble at a pressure of $0 \mathrm{MPa}$ for 90 picoseconds (ps) to melt the alloy using equilibrium MD simulations. This step is followed by rapid quenching of the alloy under the NPT ensemble at $0 \mathrm{MPa}$ with a cooling rate of $0.0038 \mathrm{~K} / \mathrm{ps}$ to reach $300 \mathrm{~K}$. We employ the Nosé-Hoover thermostat and barostat, each with a coupling time of 1 ps. Next, the structure is allowed to equilibrate for another 90 ps. A time step of $0.001 \mathrm{ps}$ is maintained throughout all our MD simulations.

The quenched alloy is, then, further equilibrated under the NPT and NVT (canonical) ensembles successively. The pressure and temperature constraints each with the coupling time of 1 ps are imposed by the Nosé-Hoover thermostat and barostat, for a total time of $90 \mathrm{ps,} \mathrm{followed} \mathrm{by} \mathrm{the} \mathrm{NVT} \mathrm{ensemble,} \mathrm{for} \mathrm{further} 90$ ps. Finally, the entire system is simulated in the absence of thermodynamic constraints for further 90 ps under the NVE (microcanonical) ensemble to ensure that we obtain an equilibrated structure. Next, tensile loading of the alloy is performed independently at $300 \mathrm{~K}$. The simulation cell is deformed in the $x$-direction of $\langle 100\rangle$ with a strain-rate of $10^{10} \mathrm{~s}^{-1}$, for the engineering strain of $0.9 \%$, while lateral boundaries are controlled using the NPT equations of motion to zero pressure. We employ the 12-6 Lennard-Jones potential with the functional details described in our earlier work [38]. The different parameters for the force field, as employed in previous MD simulations, are also available as the supplementary information [39, 40]. Each alloy examined under the CS framework underwent the structure preparation (melting, quick quenching and 
equilibration), followed by high strain deformation, as described elsewhere in details [38].

The predictive capability of the CS-MD optimization framework for multicomponent alloys is first verified for a binary Al-Fe alloy, followed by ternary and quinary combinations. The selected optimization parameters for the binary, ternary, and quinary alloys are listed in Table 1 together with the upper and lower limits of the design variable for each material. For the binary case, the $\mathrm{Al}$ elemental \% (atomic) is chosen to be the design variable, while the objective function is to increase the ultimate tensile strength (UTS) for the alloy. UTS is the maximum stress that a material can sustain within specific strain limits, which in this investigation is 0 to 90 $\%$ strain at a specified strain rate of $10^{10} \mathrm{~s}^{-1}$. The choice of the extremely-high strain rate, which is difficult to realize in typical experiments, is required to derive demonstrative predictions from MD simulations within reasonable wall times. The design space shown in Figs. 2a, c, and e has the elemental \% (atomic) of the design variable in the z-axis varying with the number of iterations and number of nests presented along the $\mathrm{y}$ and the $\mathrm{x}$ directions, respectively. The numbers of nests denote the number of solutions considered. The total number of evaluations of the objective function is the product of the number of nests and number of iterations. The predictive landscape in Figs. 2a, c and e represent the exploratory walks performed by the different nest/solutions $(=20)$ of the CS algorithm. Here, the same colored histograms represent the variation in the design parameter (atomic concentration) values with each iteration of the CS cycle. The fluctuations in the histograms during the different iterations indicate that the algorithm is employing different design values to arrive at a global optimum and achieve the desired objective function of the increased strength in the alloy. While the local search consumes about $1 / 5^{\text {th }}$ of the total search time, the rest is required for the global search when using a switching parameter of $p_{a}=0.2$. In each iteration, the complete exploration of the design variable by the different cuckoo nests analyzes the favorable Al elemental \% (atomic) that increases the strength of the binary $\mathrm{Al}_{\mathrm{x}} \mathrm{Fe}$ alloy.

The CS-MD results (Figs. $2 \mathrm{a}$ and $2 \mathrm{~b}$ ) suggest that as $\mathrm{Al} \%$ reduces the strength of the binary (Al-Fe) alloy increases from 4,000 $\mathrm{MPa}$ for $20 \% \mathrm{Al}$ to a final value of 5,000 $\mathrm{MPa}$ for $9 \% \mathrm{Al}$ in 100 objective function evaluations. Each objective function evaluation involves a complete MD computational analysis, where for a particular 
composition, a nanoscopic structure of the alloy is simulated by quenching from 2,200 $\mathrm{K}$ to $300 \mathrm{~K}$, followed by the uniform tensile deformation in the $\langle 100\rangle$ direction. The low Fe solubility in Al promotes several stable and metastable phases that often lead to the formation of a hard and brittle intermetallic with the reduced formability in rapidly-quenched $\mathrm{Al}$ alloys [41-43]. The predictions of the CS-MD approach for the high strain deformation of $\mathrm{Al}_{\mathrm{x}} \mathrm{Fe}$ alloys shows reasonable agreement with the literature, where an increase in $\mathrm{Fe} \%$ promotes higher tensile strength in quenched alloys [44-48].

Table 1: Optimization parameters used for the different alloys.

\begin{tabular}{lcccccc}
\hline Alloy composition & $\begin{array}{c}\text { Design } \\
\text { variable }\end{array}$ & $\begin{array}{c}\text { Upper } \\
\text { bound } \\
\text { (at. \%) }\end{array}$ & $\begin{array}{c}\text { Lower } \\
\text { bound } \\
(\text { at. \%) }\end{array}$ & $\begin{array}{c}\text { Switching } \\
\text { parameter } \\
\left(\mathbf{p}_{\mathbf{a}}\right)\end{array}$ & $\begin{array}{c}\text { No. } \\
\text { of } \\
\text { Nests }\end{array}$ & $\begin{array}{c}\text { No. of } \\
\text { Cycles }\end{array}$ \\
\hline Binary: AlFe & $\mathrm{Al}$ & 99 & 1 & 0.2 & 20 & 10 \\
Ternary: $\mathrm{FeNiCr}$ & $\mathrm{Fe}$ & 90 & 10 & 0.2 & 20 & 10 \\
Quinary: AlCrCoFeNi & $\mathrm{Al}$ & 99 & 1 & 0.2 & 20 & 6 \\
\hline
\end{tabular}

[Figure 2]

FIG. 2. Design-space-exploration map [panels - (a), (c), and (e)], and design variable (concentration) and objective function (UTS) variation with each cycle of CS [panels - (b), (d), and (e)], for different multicomponent alloys --- binary Al-Fe, ternary Fe$\mathrm{Ni}-\mathrm{Cr}$, and quinary $\mathrm{Al}-\mathrm{Cr}-\mathrm{Co}-\mathrm{Fe}-\mathrm{Ni}$--- examined in the CS-MD optimization framework.

The ternary $\mathrm{Fe}-\mathrm{Ni}-\mathrm{Cr}$ alloy with the design variable as the atomic $\%$ of $\mathrm{Fe}$ is next employed as a test bed for the material-optimization method. Figure $2 \mathrm{c}$ shows the design-space extent when the different cuckoos explore possible nests/solutions over the different cycles. The variations of the design parameter and objective function with each cycle of CS are shown in Fig. $2 d$ that shows an increase in the strength of the Fe-Ni-Cr alloy with the increase in the Fe content. Within 200 objective function evaluations, the algorithm is able to predict the Fe content (at. \%) that effectively raises the strength in the ternary alloy by $\sim 4 \%$. An increase in Fe content in alloys 
(binary and ternary) promotes the formation of intermetallic phases that enhance strength (tensile) in the modified alloy [42, 44, 45, 49-51].

We extend our optimization analysis to quinary alloy compositions constituted of the $\mathrm{Al}-\mathrm{Cr}-\mathrm{Co}-\mathrm{Fe}-\mathrm{Ni}$ elements. The $\mathrm{Al}$ content is the design variable, and the objective is to examine trends for the design variable that could result in a higher strength of the quinary alloy. Predictions from the design framework in 120 objective function evaluations across 6 cycles of CS runs suggest that the strength (UTS) of the quinary alloy can increase by $\sim 35 \%$. Figure $2 \mathrm{f}$ illustrates the evolution of UTS as a function of $\mathrm{Al}$ at. \% over the computed cycles. We find that reducing $\mathrm{Al}$ concentration from 10 $\%$ to $1 \%$ at the expense of increasing Fe fraction in our quinary alloy, contributes to increasing the strength of the multicomponent alloy system. The trends observed from our design framework are in good agreement with experimental measurements [52], that show an increase in UTS from $620 \mathrm{MPa}$ to $635 \mathrm{MPa}$ at $298 \mathrm{~K}$ for a decrease in $\mathrm{Al}$ $\%$ from 6.4 to 2.2 at.-\% for the $\mathrm{Al}_{\mathrm{x}} \mathrm{CrCoFeNi} \mathrm{HEA}$. We conjecture that $\mathrm{Al}$ promotes the clustering transition [38], and as its concentration reduces while that of $\mathrm{Fe}$ increases, the strength of the multicomponent alloy increases. Our predictions, which contradict the increase of strength and hardness with increasing Al content in single phase solid solutions (near equiatomic HEAs) [5], suggest that for the optimized compositions intermetallic, multi-phase or possibly amorphous structures are promoted in multicomponent alloys. It is also important to clarify that the different multi-component alloy systems considered in the study are defect-free [53, 54], and we report values (UTS) that are in the realm of ideal-strength [53, 55-57]. However, in actual materials dislocations, twinning, stacking faults, and microstructural defects, to name a few, significantly contribute to strength characteristics.

In summary, we develop and implement a combined cuckoo search and molecular dynamics based the design framework to optimize the composition of multicomponent alloys for the desired structural property, such as high tensile strength. The metaheuristic simulation algorithm is sufficiently robust to be not confined by local optimum solutions and predict the global maxima/minima. We employ the technique to examine the variation of mechanical strength under high strain-rate deformation in binary, ternary, and quinary multicomponent alloys. The results of the computational scheme reveal the correlation between the concentration of a single element (design variable) and ultimate tensile strength (objective function) 
that are qualitatively in agreement with earlier experimental measurements. The proposed technique accelerates the selection and composition of elements for a multicomponent alloy system with desired structures and properties, overcoming the limitations of trial-and-error strategies for exploring the vast materials landscape for such complex structures.

\section{Acknowledgements}

The research at Iowa State University was supported by the National Science Foundation (NSF) grant no. CMMI-1404938, the Office of Naval Research under ONR grant N00014-16-1-2548, and the High Performance Computing HPC@ISU equipment at Iowa State University, some of which has been purchased through NSF funding under MRI grant no. CNS 1229081 and CRI grant no. 1205413. We thank Dr. Ravi Kappiyoor for evaluating the robustness of the code. PKL thanks the Department of Energy (DOE), Office of Fossil Energy, National Energy Technology Laboratory (DE-FE-0008855, DE-FE-0024054, and DE-FE-001194) with Mr. V. Cedro and Mr. R. Dunst as program managers, DE-FE-001194 with the program manager Dr. J. Mullen, U.S. Army Research Office project (W911NF-13-1-0438) with the program manager Dr. D. M. Stepp, and the NSF (DMR-1611180) with the program director Dr. D. Farkas. 


\section{References}

[1] J.W. Yeh, S.K. Chen, S.J. Lin, J.Y. Gan, T.S. Chin, T.T. Shun, C.H. Tsau, S.Y.

Chang, Adv. Eng. Mater. 6(5) (2004) 299-303.

[2] Y. Zhang, T.T. Zuo, Z. Tang, M.C. Gao, K.A. Dahmen, P.K. Liaw, Z.P. Lu, Prog. Mater. Sci. 61 (2014) 1-93.

[3] M.C. Gao, J.W. Yeh, P.K. Liaw, Y. Zhang, High-Entropy Alloys: Fundamentals and Applications, Springer, Switzerland, 2015.

[4] J.W. Qiao, S.G. Ma, E.W. Huang, C.P. Chuang, P.K. Liaw, Y. Zhang, Nano-Scale Amourp. Mater. 688 (2011) 419-425.

[5] Z. Tang, M.C. Gao, H.Y. Diao, T.F. Yang, J.P. Liu, T.T. Zuo, Y. Zhang, Z.P. Lu, Y.Q. Cheng, Y.W. Zhang, K.A. Dahmen, P.K. Liaw, T. Egami, Jom 65(12) (2013) 1848-1858.

[6] L.J. Santodonato, Y. Zhang, M. Feygenson, C.M. Parish, M.C. Gao, R.J.K. Weber, J.C. Neuefeind, Z. Tang, P.K. Liaw, Nat. Comm. 6 (2015).

[7] Y. Zhang, J.W. Qiao, P.K. Liaw, J. Iron Steel Res. Int. 23(1) (2016) 2-6.

[8] A. Takeuchi, K. Amiya, T. Wada, K. Yubuta, W. Zhang, A. Makino, Entropy 15(9) (2013) 3810-3821.

[9] M.H. Tsai, Entropy 18(7) (2016).

[10] D.B. Miracle, J.D. Miller, O.N. Senkov, C. Woodward, M.D. Uchic, J. Tiley, Entropy 16(1) (2014) 494-525.

[11] J.W. Yeh, Y.L. Chen, S.J. Lin, S.K. Chen, Adv. Struct. Mater. III 560 (2007) 1-9.

[12] Y. Zhang, Y. Zhou, Pricm 6: Sixth Pacific Rim International Conference on Adv. Mater. Process., Pts 1-3 561-565 (2007) 1337-1339.

[13] A. Takeuchi, N. Chen, T. Wada, W. Zhang, Y. Yokoyama, A. Inoue, J.W. Yeh, Procedia Eng. 36 (2012) 226-234.

[14] Y. Zhang, X. Yang, P.K. Liaw, Jom 64(7) (2012) 830-838.

[15] J.W. Yeh, Jom 65(12) (2013) 1759-1771.

[16] C. Zhang, F. Zhang, H. Diao, M.C. Gao, Z. Tang, J.D. Poplawsky, P.K. Liaw, Mater. Des. 109 (2016) 425-433.

[17] R. Feng, M. Gao, C. Lee, M. Mathes, T. Zuo, S. Chen, J. Hawk, Y. Zhang, P. Liaw, Entropy 18(9) (2016) 333.

[18] T.K. Sharma, M. Pant, M. Singh, Mater. Manuf. Process 28(7) (2013) 788-802.

[19] S.E.K. Fateen, A. Bonilla-Petriciolet, Sci. World J. (2014). 
[20] M.N. Shehata, S.E.K. Fateen, A. Bonilla-Petriciolet, Fluid Phase Equilibr. 409 (2016) 280-290.

[21] Udayraj, K. Mulani, P. Talukdar, A. Das, R. Alagirusamy, Int. J. Heat Mass Trans. 89 (2015) 359-378.

[22] S.H. Xu, J.P. Liu, F.H. Zhang, L. Wang, L.J. Sun, Sensors 15(9) (2015) 2103321053.

[23] C.J. Lin, S.C. Su, Int. J. Fuzzy Syst. 13(2) (2011) 140-147.

[24] T.D. Liu, L.Y. Xu, G.F. Shao, N.N. Tu, J.P. Tao, Y.H. Wen, J. Alloys Comp. 663 (2016) 466-473.

[25] A. Jenab, I.S. Sarraf, D.E. Green, T. Rahmaan, M.J. Worswick, Mater. Design 94 (2016) 262-273.

[26] M.A. Khansary, A.H. Sani, Fluid Phase Equilibr. 365 (2014) 141-145.

[27] T. Ishikawa, T. Oda, N. Suzuki, K. Shimizu, High Press. Res. 35(1) (2015) 3741.

[28] H.T. Xie, J.S. Zhao, Q.B. Wang, Y.Y. Sui, J.K. Wang, X.M. Yang, X.D. Zhang, C. Liang, Sci. Rep. 5 (2015).

[29] C. Brandt, G. Fieg, X. Luo, Heat Mass Trans. 47(8) (2011) 1019-1026.

[30] J.Z. Wang, H. Jiang, Y.J. Wu, Y. Dong, Energy 81 (2015) 627-644.

[31] X.S. Yang, S. Deb, Neural Comput. Appl. 24(1) (2014) 169-174.

[32] M.Jamil, H.J. Zepernick, X.S. Yang, IEEE Military Communications Conference (Milcom), San Diego, CA (2013) 823-828.

[33] L.D. Coelho, C.E. Klein, S.L. Sabat, V.C. Mariani, Energy 75 (2014) 237-243.

[34] X.S. Yang, S. Deb, Wor. Cong. Nat. Biol. (2009) 210-214.

[35] A. Natarajan, S. Subramanian, K. Premalatha, Int. J. Bio-Inspir. Comp. 4(2) (2012) 89-99.

[36] X.-S. Yang, Nature-inspired optimization algorithm, First ed., Elsevier, MA, USA, 2014.

[37] S. Plimpton, J. Comp. Phys. 117 (1995) 1-19.

[38] A. Sharma, P. Singh, D.D. Johnson, P.K. Liaw, G. Balasubramanian, Sci. Rep. 6 (2016).

[39] L. Xie, P. Brault, A.L. Thomann, J.M. Bauchire, Appl. Surf. Sci. 285P (2013) 810-816.

[40] D.B. Graves, P. Brault, Journal of Physics D: Appl. Phys. 42(194011) (2009) 127. 
[41] J.M. Cubero-Sesin, Z. Horita, Metall. Mater. Trans. A 43a(13) (2012) 51825192.

[42] N.A. Belov, A.A. Aksenov, D.G. Eskin, Iron in aluminum alloys: impurity and alloying element, Taylor \& Francis, London, 2002.

[43] A. Lendvai, J. Mater. Sci. Lett. 5(12) (1986) 1219-1220.

[44] S.D. Kaloshkin, V.V. Tcherdyntsev, I.A. Tomilin, D.V. Gunderov, V.V. Stolyarov, Y.V. Baldokhin, I.G. Brodova, E.V. Shelekhov, Mater. Trans. 43(8) (2002) 2031-2038.

[45] V.V. Tcherdyntsev, S.D. Kaloshkin, E.A. Afonina, I.A. Tomilin, Y.V. Baldokhin, E.V. Shelekhov, D.V. Gunderov, I.G. Brodova, V.V. Stolyarov, Defect Diffus. Forum 216-2 (2003) 313-321.

[46] D.K. Mukhopadhyay, C. Suryanarayana, F.H. Froes, Metall. Mater. Trans. A 26(8) (1995) 1939-1946.

[47] F. Cardellini, V. Contini, G. Mazzone, J. Mater. Sci. 31(16) (1996) 4175-4180.

[48] F. Cardellini, V. Contini, R. Gupta, G. Mazzone, A. Montone, A. Perin, G. Principi, J. Mater. Sci. 33(10) (1998) 2519-2527.

[49] J.S. Wrobel, N.M. Duc, M.Y. Lavrentiev, M. Muzyk, S.L. Dudarev, Phys. Rev. B 91(2) (2015).

[50] D.J. Skinner, K. Okazaki, Scripta Metall. Mater. 18(9) (1984) 905-909.

[51] B.R. Kumar, S. Sharma, P. Munda, R.K. Minz, Mater. Design 50 (2013) 392398.

[52] D.Y. Li, Y. Zhang, Intermet. 70 (2016) 24-28.

[53] X.Q. Li, S. Schonecker, J.J. Zhao, B. Johansson, L. Vitos, Phys. Rev. B 87(21) (2013).

[54] R. Komanduri, N. Chandrasekaran, L.M. Raff, Int. J. Mech. Sci. 43(10) (2001) 2237-2260.

[55] A. Breidi, S.G. Fries, A.V. Ruban, Phys. Rev. B 93(14) (2016).

[56] M. Hara, Y. Shimizu, T. Yano, N. Takesue, T. Furuta, S. Kuramoto, Int. J. Mater. Res. 100(3) (2009) 345-348.

[57] V. Van Hung, K. Masuda-Jindo, N.T. Hoa, J. Mater. Res. 22(8) (2007) 22302240. 


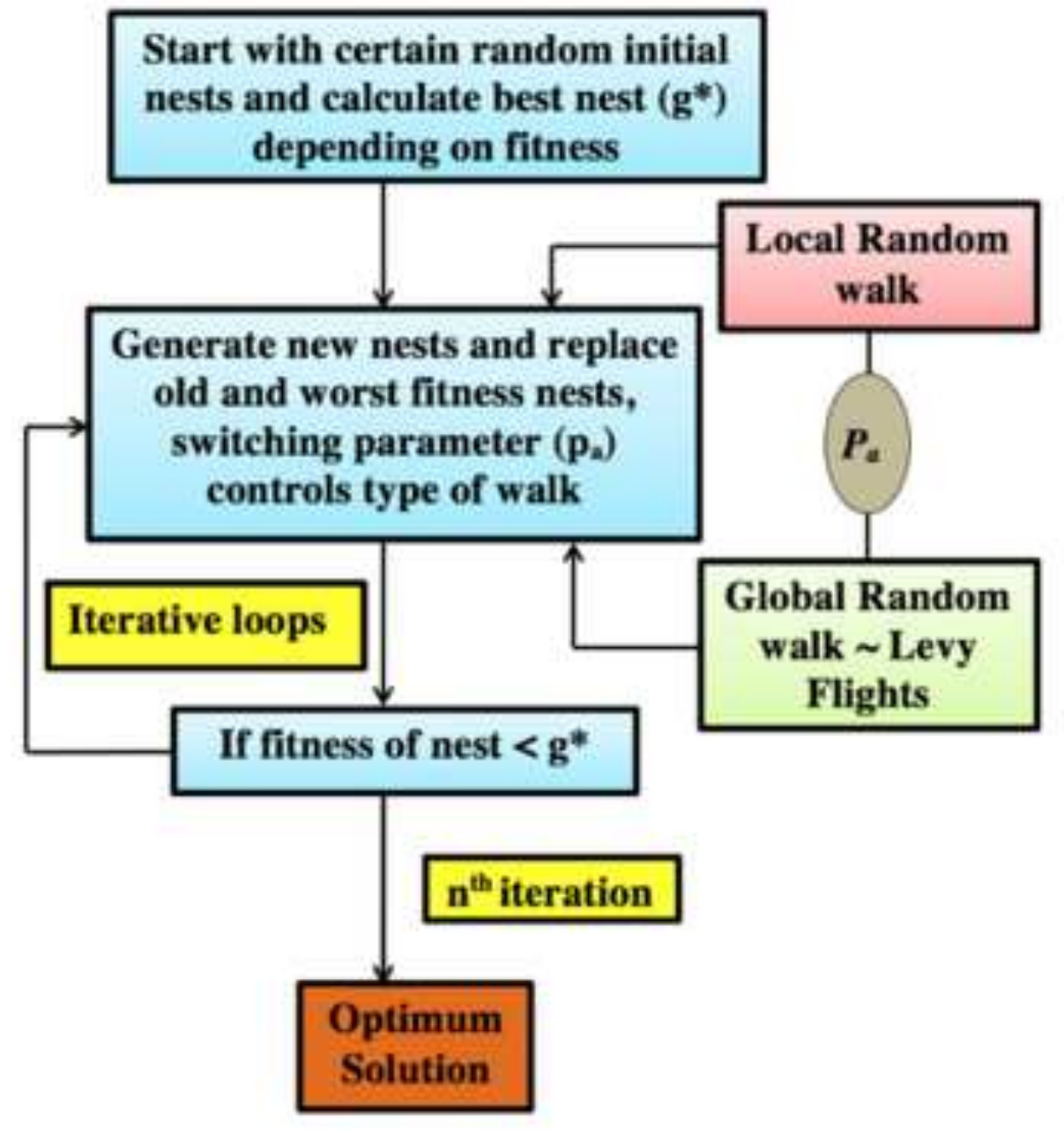

(a)

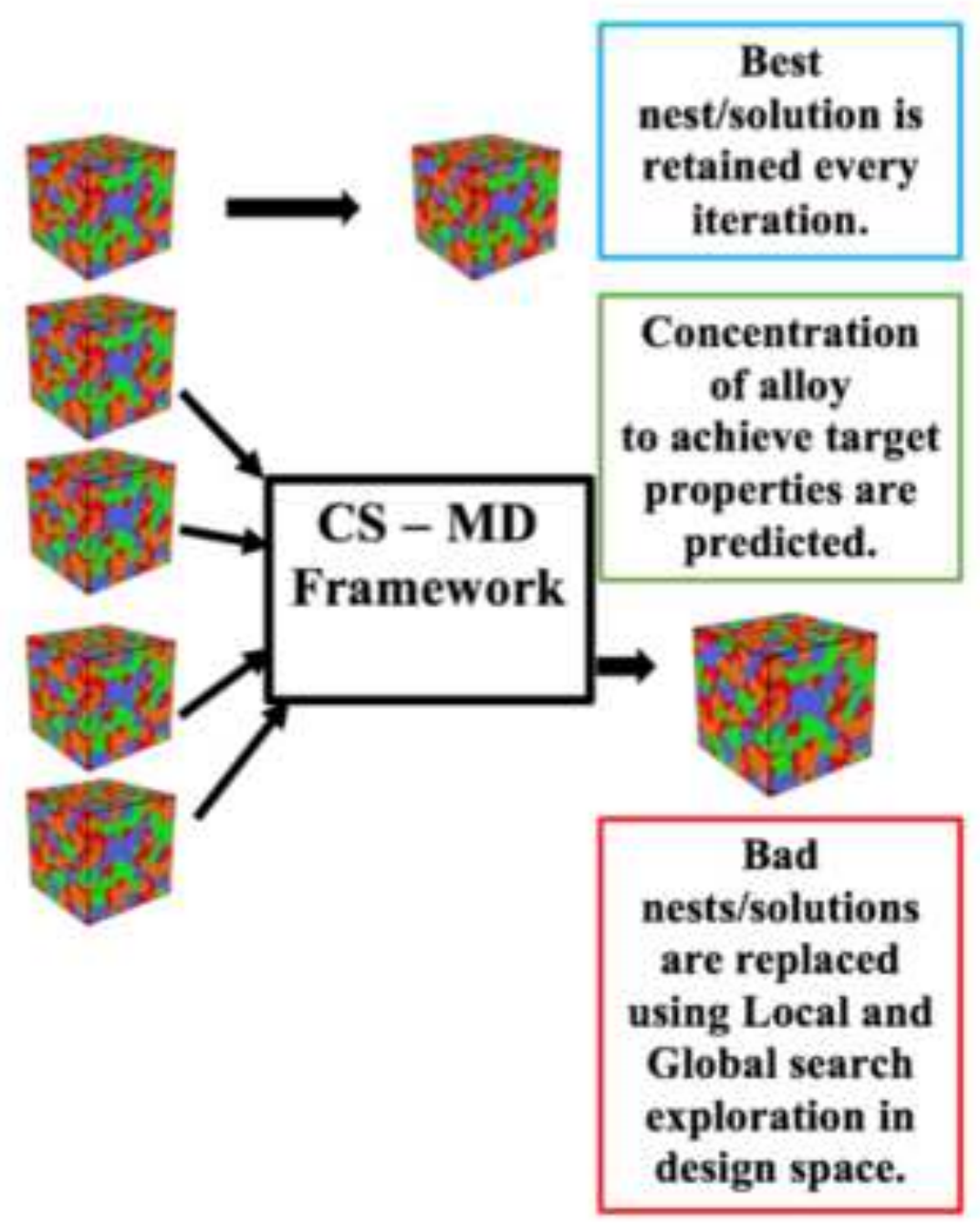

(b) 


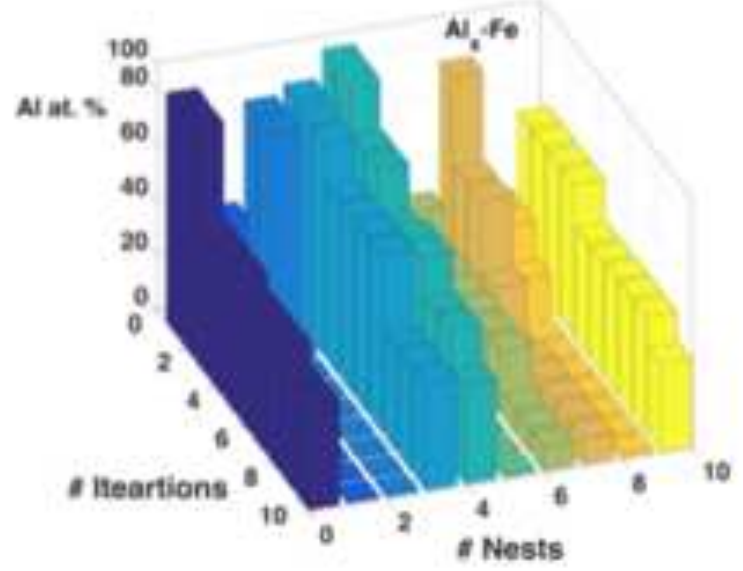

(a)

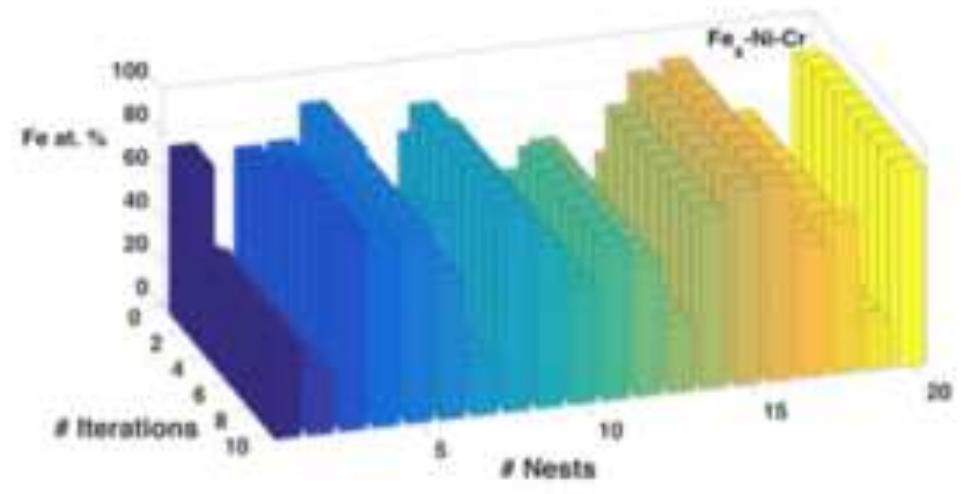

(c)

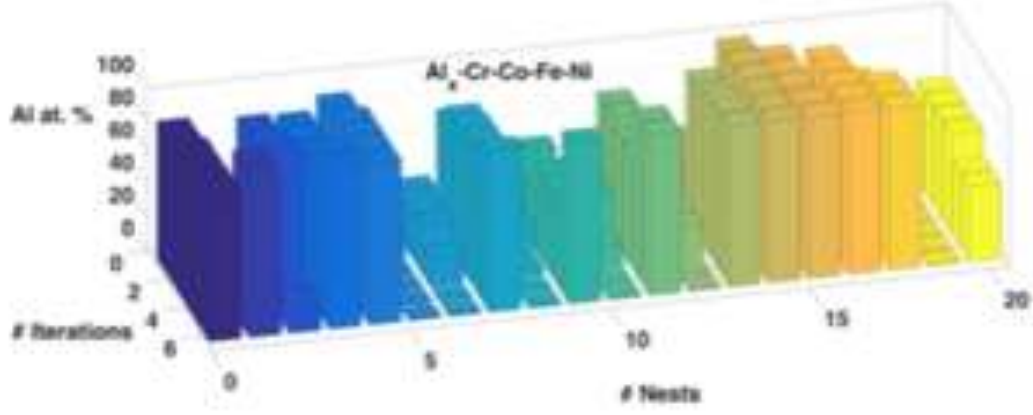

(e)

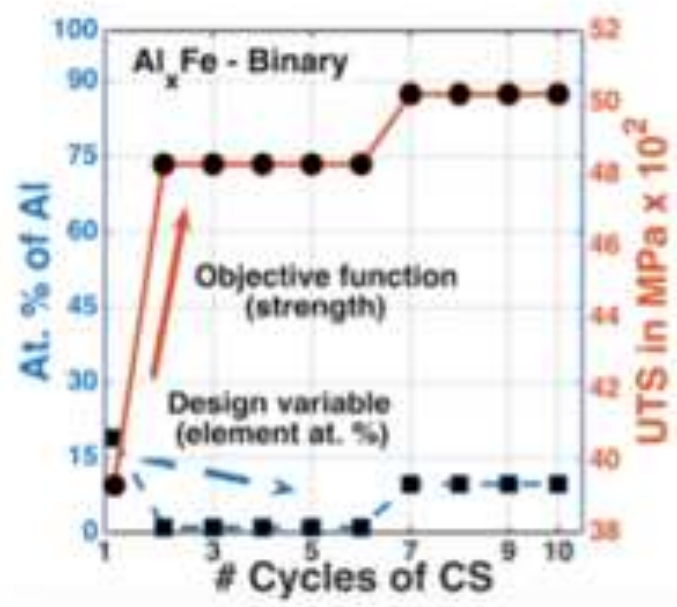

(b)

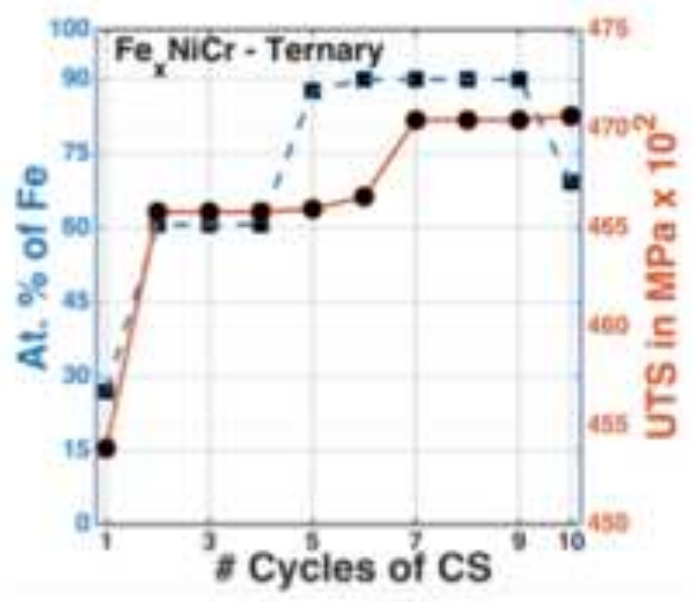

(d)

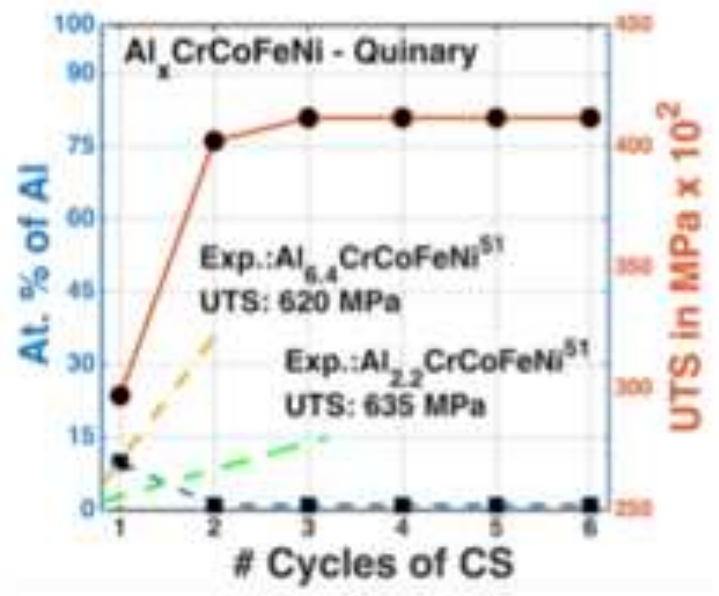

(f) 


\section{${ }^{*}$ Graphical Abstract}
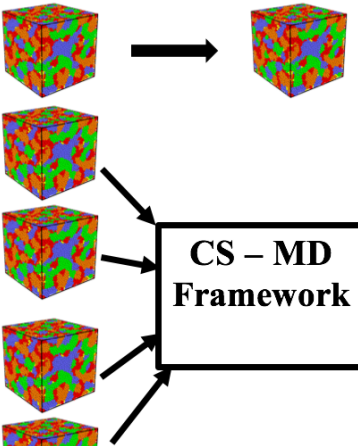

\section{Best}

nest/solution is retained every iteration.

\section{Concentration}

\section{of alloy}

to achieve target properties are predicted.
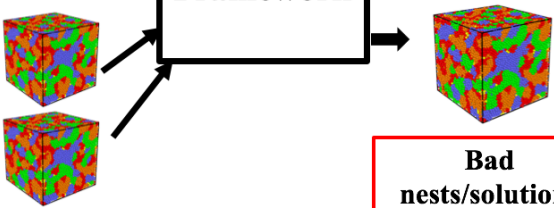

\section{Bad}

nests/solutions are replaced using Local and Global search exploration in design space. 